\title{
Pointwise Bounds in the Cauchy Problem of Elastic Plates
}

\author{
L. E. Payne*
}

(November 3, 1960)

\begin{abstract}
Let an elastic plate occupy a region $D$ with boundary $C$. On a portion $\Sigma$ of $C$ the deflection, slope, moment, and shear are measured. In terms of these data, and crude bounds for the maximum deflection and moment, pointwise bounds for the value of the deflection and its derivatives in $D$ are obtained. These bounds are such that if the data on $\Sigma$ and the loading perpendicular to the plane of the plate tend to zero, the deflection tends to zero at every point in $D$. Similar bounds are obtained in the slow flow problem for a viscous fluid.
\end{abstract}

\section{Introduction}

The following plate problem is considered. Let an elastic plate occupying a region $D$ in the $x y$-plane be subjected to a load $p(x, y)$ normal to the plane of the plate. The deflection $v$ satisfies the equation

$$
\Delta^{2} v=\left(\frac{\partial^{4} v}{\partial x^{4}}+2 \frac{\partial^{4} v}{\partial x^{2} d y^{2}}+\frac{\partial^{4} v}{\partial y^{4}}\right)=p / \mathscr{D}
$$

where $D$ denotes the plate rigidity (see [3, p. 488]). ${ }^{1}$ We assume that on a portion $\Sigma$ of the boundary of $D$ we are able to measure the deflection, slope, the sum of the two principle moments, and the normal component of shear with known error; or equivalently we assume

$$
\left.\begin{array}{ll}
\int_{\Sigma}(v-f)^{2} d \sigma \leq \alpha_{1}, & \int_{\Sigma}\left(v_{, i}-F_{i}\right)\left(v_{, i}-F_{i}\right) d \sigma \leq \alpha_{2} \\
\int_{\Sigma}(\Delta v-g)^{2} d \sigma \leq \alpha_{3}, & \int_{\Sigma}\left(\Delta v_{, i}-G_{i}\right)\left(\Delta v_{, i}-G_{i}\right) d \sigma \leq \alpha_{4}
\end{array}\right\}
$$

where $f, F_{i}, g$, and $G_{i}$ are the measured values of $v, v,,_{i}, \Delta v$, and $\Delta v,_{i}$ respectively. In (1.2) the comma denotes differentiation and a repeated subscript $i$ implies summation over $i(i$ $=1,2)$. The $\Delta$ symbol is used throughout to denote the Laplace operator.

In (1.2) then the $\alpha_{i}$ are assumed to be small known constants. We wish to obtain upper and lower bounds for the solution $v$ at any point in $R$.

It is well known that as it stands the Cauchy problem for the biharmonic equation is not well posed (see Hadamard [1]). The solution does not depend in a continuous way on the boundary data. We leave aside the question of existence of solution and merely remark that in most physical situations in which one would be interested in considering such a problem the question does not arise. For instance, we might wish to treat an ordinary plate boundary value problem, but find that part of the boundary is inaccessible for measurement of boundary data whereas on another portion of the boundary it is possible to measure $v$, $\frac{\partial v}{\partial n}, \Delta v$, and $\frac{\partial}{\partial n}(\Delta v)$.

If in addition to the Cauchy data we have uniform bounds for both $v$ and $\Delta v$ throughout $D$ then the solution to the plate problem will be seen to be stable. This is in fact an immediate consequence of the results of Laurentiev [2].

*University of Maryland; consultant, National Bureau of Standards.

${ }^{1}$ Figures in brackets indicate the literature references at the end of this paper. 
In order to simplify the procedure somewhat we decompose the solution $v$ into

$$
v=v_{p}+v^{\prime},
$$

where $v_{p}$ is any particular solution of (1.1) defined in $D$ and $v^{\prime}$ satisfies

$$
\Delta^{2} v^{\prime}=0
$$

in $D$. We approximate $v^{\prime}$ by a biharmonic function $\varphi$ and set $w=v^{\prime}-\varphi$. Thus $w$ satisfies in $D$

$$
\Delta^{2} w=0
$$

Also bounds for the Cauchy expressions

$$
\left.\begin{array}{ll}
\eta_{1}=\int_{\Sigma} w^{2} d \sigma, & \eta_{2}=\int_{\Sigma} w_{, i} w_{, i} d \sigma \\
\epsilon_{1}=\int_{\Sigma}(\Delta w)^{2} d \sigma, & \epsilon_{2}=\int_{\Sigma} \Delta w_{, i} \Delta w_{, i} d \sigma
\end{array}\right\}
$$

are known. In fact

$$
\eta_{1}=\int_{\Sigma}\left[\left(v^{\prime}+v_{p}-f\right)-\left(\phi+v_{p}-f\right)\right]^{2} d \sigma \leq 2 \alpha_{1}+2 \int_{\Sigma}\left(\phi+v_{p}-f\right)^{2} d \sigma
$$

with similar inequalities for $\eta_{2}, \epsilon_{1}$, and $\epsilon_{2}$. It is clear that if $\alpha_{1}$ is small and $\phi$ can be chosen to approximate $f-v_{p}$ in mean square on $\Sigma$ then $\eta_{1}$ will be small. A similar statement holds for $\eta_{2}$, $\epsilon_{1}$, and $\epsilon_{2}$. We shall show in this paper that it is possible to obtain an a priori bound for $w$ of the following type at any point in $R$ :

$$
|w(P)|^{2} \leq A_{1} M_{1}^{1-\delta_{1}}\left\{a_{1} \eta_{1}+a_{2} \eta_{2}\right\}^{\delta_{1}}+A_{2} M_{2}^{1-\delta_{2}}\left\{b_{1} \epsilon_{1}+b_{2} \epsilon_{2}\right\}^{\delta_{2}},
$$

where $A_{1}, A_{2}, a_{1}, a_{2}, b_{1}, b_{2}$, are determined constants, $\delta_{1}$ and $\delta_{2}$ are constants satisfying

$$
0<\delta_{2}<\delta_{1}<1,
$$

and $M_{1}$ and $M_{2}$ are the uniform bounds for $w$, and $\Delta w$ in $D$. It is clear that knowledge of uniform bounds for $v^{\prime}$ and $\Delta v^{\prime}$ implies knowledge of uniform bounds for $w$ and $\Delta w$ since $w-v^{\prime}$ is a known function.

With an inequality of type (1.8) it is clear from (1.7) and the corresponding inequalities for $\eta_{2}, \epsilon_{1}$, and $\epsilon_{2}$ that if the $\alpha_{i}$ are sufficiently small and the Rayleigh-Ritz technique is used to make the integral expression on the extreme right small then we obtain close upper and lower bounds for $v^{\prime}(P)$. This paper will be concerned then with the establishing of (1.8).

\section{A priori Bounds}

We consider a two-dimensional region $D$ bounded by a closed surface $C$. On a portion $\Sigma$ of $C$, Cauchy data are prescribed. We denote the remainder of the surface $(C-\Sigma)$ by $\bar{\Sigma}$. (As will become apparent later the portion $\bar{\Sigma}$ need not actually be prescribed.) We now choose the origin of a polar coordinate system at a point $P_{0}$ which has the property that there exists a circle $K(r)$ of radius $r$ and center at $P_{0}$ such that the intersection of $C$ and $K(r)$ contains no point of $\bar{\Sigma}$. (If two or more disjoint regions are formed by the intersection of $K(r)$ and $D$, it is sufficient that the boundary of any one of these regions contains no points of $\bar{\Sigma}$.) We denote by $D_{r}$ the intersection of $K(r)$ and $D$ (the portion whose boundary contains no points of $\bar{\Sigma}$ if two or more disjoint regions are formed) and require that $P_{0}$ lie outside of $D_{r}$. We denote by $R$ the radius of the largest circle satisfying the above conditions (for a fixed $P_{0}$ ) and by $r_{0}$ the radius of the smallest such circle. As indicated in the previous paper [5] we locate $P_{0}$ in such a 
way that $K\left(r_{0}\right)$ is tangent to $D$ at some point of $\Sigma$. Finally we denote by $\omega(r)$ the portion of the surface of $K(r)$ which lies interior to $C$.

As in [5] we introduce for any function $u$ the notation

$$
\left.\begin{array}{l}
\epsilon_{1}=\int_{\Sigma} u^{2} d \sigma \\
\epsilon_{2}=\int_{\Sigma}\left[\left(\frac{\partial u}{\partial x}\right)^{2}+\left(\frac{\partial u}{\partial y}\right)^{2}\right] d \sigma,
\end{array}\right\}
$$

where $d \sigma$ denotes an element of surface area on $\Sigma$, and let

$$
A_{r}(u)=\iint_{D_{r}}\left[\left(\frac{\partial u}{\partial x}\right)^{2}+\left(\frac{\partial u}{\partial y}\right)^{2}\right] d x d y .
$$

In [5] we proved that if $u$ satisfies

then the functional

$$
\Delta u=0, \quad|u| \leq M \text { in } D,
$$

$$
v(r, u)=\log \left[\int_{r_{0}}^{r} \rho^{-1} A_{\rho}(u) d \rho+\kappa_{1} \epsilon_{1}+\kappa_{2} \epsilon_{2}\right]+C(\log r)^{2}
$$

is a convex functional of $\log r$ in $r_{0}<r<R$, where

$$
C=\frac{1}{2}(1+\sqrt{2}) r_{0} R^{-1} ; \quad \kappa_{1}=\frac{3}{2} r_{0}^{-1} ; \quad \kappa_{2}=\frac{1}{2}\left\{r_{0}^{2}\left[\log \left(R / r_{0}\right)\right]^{2}+R^{2}\right\} r_{0}^{-1} .
$$

It was emphasized in [5] that the constants in [2.5] are usually not the optimal constants, which in any specific problem depend on the geometry of $\Sigma$ and $D$ as well as the location of $P_{0}$.

An evaluation of the integral in (2.4) leads immediately to the inequality

$$
\left|\int_{r_{0}}^{r} \rho^{-1} A_{\rho}(u) d \rho-\frac{1}{2} r^{-1} \int_{\omega(r)} u^{2} d s\right| \leq r_{0}^{-1} \epsilon_{1}+r_{0} \log \left(R / r_{0}\right) \epsilon_{2} .
$$

Following the procedure indicated in [5] we are lead to the inequality

$$
r^{-1} \int_{\omega(r)} u^{2} d s-+r_{0}^{-1} \epsilon_{1}+R^{2} r_{0}^{-1} \epsilon_{2} \leq 2 B M^{2(1-\delta)}\left(\kappa_{1} \epsilon_{1}+\kappa_{2} \epsilon_{2}\right)^{\delta} .
$$

The constant $B$ is easily computed and in this case

$$
\delta=\log (R / r) / \log \left(R / r_{0}\right) .
$$

In the plate problem under consideration (1.5), (1.6) we now set

$$
\Delta w=u
$$

Then (2.7) is valid with $u$ replaced by $\Delta w$. Since we shall need it later we obtain now by integration the following inequality

$$
\begin{aligned}
\iint_{D_{r}}(\Delta w)^{2} d x d y+\frac{1}{2}\left(r^{2}-r_{0}^{2}\right) r_{0}^{-1} \epsilon_{1}+\frac{1}{2}\left(r^{2}-r_{0}^{2}\right) R^{2} r_{0}^{-1} \epsilon_{2} \\
\leq 2 B \frac{\left[r^{2} M^{2(1-\delta)}\left(\kappa_{1} \epsilon_{1}+\kappa_{2} \epsilon_{2}\right)^{\delta}\right]\left[1-\frac{r_{0}^{2}}{r^{2}}\left(\frac{\kappa_{1} \epsilon_{1}+\kappa_{2} \epsilon_{2}}{M^{2}}\right)^{1-\delta}\right] \log \left(R / r_{0}\right)}{\log \left[M^{2} R^{2} / r_{0}^{2}\left(\kappa_{1} \epsilon_{1}+\kappa_{2} \epsilon_{2}\right)\right]} .
\end{aligned}
$$

Note that for

$$
\kappa_{1} \epsilon_{1}+\kappa_{2} \epsilon_{2} \leq M^{2}
$$


which must hold if the estimates are to be useful, we obtain the cruder but simpler inequality

$$
\iint_{D_{r}}(\Delta w)^{2} d s d y+\frac{1}{2}\left(r^{2}-r_{0}^{2}\right)^{-1} r_{0} \epsilon_{1}+\frac{1}{2}\left(r^{2}-r_{0}^{2}\right) R^{2} r_{0}^{-1} \epsilon_{2} \leq B r^{2} M^{2(1-\delta)}\left(\kappa_{1} \epsilon_{1}+\kappa_{2} \epsilon_{2}\right)^{\delta} .
$$

We wish to find a bound for $\int_{\omega(r)} w^{2} d s$. But once such a bound is known then pointwise bounds for $w$ at points in $D_{r}$ follow at once; for we need merely introduce the biharmonic Green's function $\Gamma(P, Q)$ which vanishes with its Laplacian on the circle of radius $r$ and center at $P_{0}$, and has a fundamental singularity at a point $P$ in $D_{r}$. We then make use of the identity

$w(P)=-\int_{\omega(r)} w \frac{\partial}{\partial n}(\Delta \Gamma) d s-\int_{\omega(r)} \Delta w \frac{\partial \Gamma}{\partial n} d s-\int_{\Sigma^{\prime}}\left[w \frac{\partial}{\partial n}(\Delta \Gamma)-\frac{\partial w}{\partial n} \Delta \Gamma+\Delta w \frac{\partial \Gamma}{\partial n}-\frac{\partial}{\partial n}(\Delta w) \Gamma\right] d \sigma$,

where $\Sigma^{\prime}$ denotes the portion of $\Sigma$ which lies interior to $K(r)$. An application of Schwarz's. inequality then yields the desired result.

In order to obtain the bound for $\int_{\omega(r)} w^{2} d s$ we introduce the following functional:

$$
U(r, w)=\log \left\{\int_{r_{0}}^{r} \rho^{-p}\left[A_{\rho}(w)+\iint_{D_{\rho}} w \Delta w d x d y\right] d \rho+l_{1} \eta_{1}+l_{2} \eta_{2}+l_{3} \eta_{3}+l_{4} \eta_{4}\right\}+C_{1} r^{-2 q},
$$

where

$$
\left.\begin{array}{l}
\eta_{1}=\int_{\Sigma} w^{2} d \sigma, \quad \eta_{2}=\int_{\Sigma} w,_{i} w,_{i} d \sigma, \\
\eta_{3}=\int_{D_{R_{1}}} \int_{(\Delta w)^{2} d x d y, \eta_{4}=2 B R_{1} M^{2(1-\beta)}\left(\kappa_{1} \epsilon+\kappa_{2} \epsilon_{2}\right)^{\beta}}
\end{array}\right\}
$$

for

and

$$
r_{0}<r<R_{1}<R ; \quad \beta=\log \left(R / R_{1}\right) / \log \left(R / r_{0}\right)
$$

$$
p>1, \quad q>p-1 .
$$

We demonstrate that for $p$ and $q$ satisfying $(2.17)$ and the constants $l_{1}, l_{2}, l_{3}, l_{4}$, and $C_{1}$ suitably chosen, $U$ is a convex function of $r^{-i}$.

To this end we introduce the notation

$$
U(r, w)=\log E(r, w)+C_{1} r^{-2 q}
$$

(where $E$ is the term in braces in (2.14)), and form

$$
\begin{aligned}
q^{2} E^{2} \frac{\partial^{2} U}{\left(\partial r^{-q}\right)^{2}} & =E\left\{(q+1-p) r^{2 q+1-p}\left[A_{r}(w)+\iint_{D_{r}} w \Delta w d x d y\right]+r^{2(q+1)-p}\left(\int _ { \omega ( r ) } \left[|\operatorname{grad} w|^{2}\right.\right.\right. \\
& +w \Delta w] d s)\}-r^{2(q+1-p)}\left[A_{r}(w)+\iint_{D_{r}} w \Delta w d x d y\right]^{2}+2 q^{2} C_{1} E^{2}
\end{aligned}
$$

As in [5] we obtain in a straightforward manner the inequality

$$
m_{1} \eta_{1}+m_{2} \eta_{2} \leq E-\frac{1}{2} r^{-p} \int_{\omega(r)} w^{2} d s-\frac{1}{2}(p-1) \iint_{D_{r}} \rho^{-(p+1)} w^{2} d x d y-l_{3} \eta_{3}-l_{4} \eta_{4} \leq n_{1} \eta_{1}+n_{2} \eta_{2},
$$


where

$$
\left.\begin{array}{ll}
m_{1}=l_{1}-\frac{(p+2) r_{0}^{-p}}{2(p+1)}, & m_{2}=l_{2}-\frac{r_{0}-(p-2)}{2(p+1)}, \\
n_{1}=l_{1}+\frac{(p+2) r_{0}^{-p}}{2(p+1)}, & n_{2}=l_{2}+\frac{r_{0}{ }^{-(p-2)}}{2(p+1)} \cdot
\end{array}\right\}
$$

The constants $l_{1}$ and $l_{2}$ are to be so chosen that $m_{1}$ and $m_{2}$ are positive.

By Green's theorem

$$
A_{r}(w)+\iint_{D_{r}} w \Delta w d x d y=\int_{w(r)} w \frac{\partial w}{\partial r} d s+\int_{\Sigma^{\prime}} w \frac{\partial w}{\partial n} d \sigma .
$$

Thus by applying Schwarz's inequality in (2.22) and using the fact that the arithmetic mean is not less than the geometric mean we obtain from (2.19) using (2.21), (2.7), and (2.9)

$$
\begin{aligned}
q^{2} E^{2} \frac{\partial^{2} U}{\left(\partial r^{-q}\right)^{2}} \geq & \frac{1}{2} r^{-p} \int_{\omega(r)} w^{2} d s\left\{(q+1-p) r^{2 q+1-p}\left[A_{r}(w)-\frac{b_{1}}{2} r^{-2} \iint_{D_{r}} w^{2} d x d y-\frac{r^{2}}{2 b_{1}} \eta_{3}\right]\right. \\
& \left.+r^{2(q+1)-p}\left[\int_{\omega(r)}\left\{\left(\frac{\partial w}{\partial s}\right)^{2}-\left(\frac{\partial w}{\partial r}\right)^{2}\right\} d s-\frac{b_{2} r^{-2}}{2} \int_{\omega(r)} w^{2} d s-\frac{r^{2}}{2 b_{2}} \eta_{4}\right]-\frac{\alpha_{1}}{2}\left(\gamma_{1} \eta_{1}+\gamma_{2} \eta_{2}\right)\right\} \\
& +\left[\frac{(p-1)}{2} \iint_{D_{r}} r^{-(p+1)} w^{2} d x d y+m_{1} \eta_{1}+m_{2} \eta_{2}+l_{3} \eta_{3}+l_{4} \eta_{4}\right] \\
& \times\left\{(q+1-p) r^{2 q+1-p}\left[A_{r}(\omega)-\frac{b_{3} r^{-2}}{2} \iint_{D_{r}} w^{2} d x d y-\frac{r^{2}}{2 b_{3}} \eta_{3}\right]\right. \\
& \left.+\frac{1}{2 \alpha_{1}} \int_{\omega(r)}\left(\frac{\partial u}{\partial r}\right)^{2} d s\left(\gamma_{1}(q+1)-p\left[\int_{\omega(r)} \mid \operatorname{grad} \eta_{1}+\gamma_{2} \eta_{2}\right)-\eta_{1} \eta_{2}-\frac{b_{4}}{2} \int_{\omega(r)} w^{2} d s-\frac{\eta_{4}}{2 b_{4}}\right]\right\} \\
& +2 q^{2} C_{1}\left\{\frac{r^{-p}}{2} \int_{\omega(r)} w^{2} d s+\frac{(p-1)}{2} \iint_{D_{r}} r^{-(p+1)} w^{2} d x d y+l_{3} \eta_{3}+l_{4} \eta_{4}+m_{1} \eta_{1}+m_{2} \eta_{2}\right\}^{2} .
\end{aligned}
$$

where $\alpha_{1}, b_{1}, b_{2}, b_{3}$, and $b_{4}$ are arbitrary positive constants. From the Rellich identity [6, 7], it follows that

$r \int_{\omega(r)}\left\{\left(\frac{\partial w}{\partial s}\right)^{2}-\left(\frac{\partial w}{\partial r}\right)\right\} d s=2 \iint_{D_{r}} r \frac{\partial w}{\partial r} \Delta w d x d y+2 \int_{\Sigma^{\prime}} r \frac{\partial r}{\partial \sigma} \frac{\partial w}{\partial \sigma} \frac{\partial w}{\partial n} d \sigma-\int_{\Sigma^{\prime}} r \frac{\partial r}{\partial n}|\operatorname{grad} w|^{2} d \sigma$.

Thus, for any $\delta_{1} 0$,

$$
\int_{\omega(r)}\left\{\left(\frac{\partial w}{\partial s}\right)^{2}-\left(\frac{\partial w}{\partial r}\right)^{2}\right\} d s \geq-\frac{\delta_{1}}{r} A_{r}(w)-\frac{r}{\delta_{1}} \eta_{3}-\sqrt{2} \eta_{2} .
$$

If $\partial^{2} U /\left(\partial r^{-q}\right)^{2}$ is to be non-negative, then the coefficient of the term $A_{r}(w) \int_{\omega(r)} w^{2} d s$ must be non-negative. After insertion of (2.25) in (2.23) we find that this coefficient is positive if

$$
q-p+1-\delta_{1} \geq 0
$$


The coefficients of all other terms may be made positive by choosing $C_{1}$ sufficiently large provided

$$
p>1 \text {. }
$$

But (2.26) and (2.27) are just the imposed conditions (2.17). Hence for such a choice of the constants, $U$ is a convex function of $r^{-q}$.

We now let

It follows then that

$$
\delta^{*}=\left[\left(R_{1} / \gamma_{0}\right)^{-q}-1\right] /\left[\left(R_{1} / \gamma_{0}\right)^{-q}-1\right]
$$

$$
e^{C r}{ }^{-2 q} E(r, w) \leq\left[e^{C R_{1}-2 q} E\left(R_{1}, w\right)\right]^{1-\delta^{*}}\left[e^{C r_{0}-2 q} E\left(r_{0}, w\right)\right]^{\delta^{*}}
$$

Using (2.20) we obtain the inequality

$$
\begin{aligned}
\frac{1}{2} r^{-p} \int_{\omega(r)} w^{2} d s+\frac{(p-1)}{2} \int w^{2} r^{-(p+1)} d x d y & +m_{1} \eta_{1}+m_{2} \eta_{2}+l_{3} \eta_{3}+l_{4} \eta_{4} \\
& \leq C_{1} M_{1}^{1-\delta^{*}}\left\{m_{1} \eta_{1}+m_{2} \eta_{2}\right\}^{\delta^{*}}+C_{2} M_{2}^{1-\delta^{\prime}}\left\{\kappa_{1} \epsilon_{1}+\kappa_{2} \epsilon_{2}\right\}^{\delta^{\prime}}
\end{aligned}
$$

where the constants $C_{1}$ and $C_{2}$ are determined as indicated in [5]. We have used (2.10) or (2.12) for $\eta_{3}$ and the notation

$$
\delta^{\prime}=\delta \delta^{*}
$$

By use of Schwarz's inequality and inequalities (2.7), (2.9), and (2.30), equation (2.13) then yields the desired pointwise bounds for $w(P)$ at any point $P$ in $D_{r}$.

In order to obtain the desired bounds in $D-D_{r}$ we first form from $(2.13) w,{ }_{i}, \Delta w$ and $\Delta w,{ }_{i}$. For $P$ a finite distance from the boundary of $D_{r}$ the differentiation may be carried out under the integral sign on the right and all of the resulting integrals converge. Thus using (2.7), (2.9), (2.13), (2.29) we obtain the pointwise bounds in $D_{r}$ not only for $w(P)$, but also for the quantities $|\operatorname{grad} w|, \Delta w$ and grad $\Delta w$ (in fact estimates for any derivative of $w$ ). If we now integrate these inequalities over a portion of a spherical surface $\omega\left(r_{1}\right)$, where $r_{1}<r$ and each point on $\omega\left(r_{1}\right)$ is a finite distance from $\Sigma$, then we may regard $\omega\left(r_{1}\right)$ as a new Cauchy surface and proceed to define a new region $D_{r_{1}}$, continuing as indicated in [5]. We are thus able to obtain pointwise bounds for $w$ and its derivatives at any point on the interior of $D$.

\section{Analogous Viscous Flow Problems}

Instead of interpreting our biharmonic Cauchy problem as a plate problem we could also have considered $v$ in (1) to be the stream function in the slow flow of an incompressible viscous fluid [4, p. 541] (here $p \equiv 0$ ). However, in this problem the actual value of $v$ is of no physical significance. The velocity components are expressed in terms of derivatives of $v$, and the physical quantities which we measure on $\Sigma$ are the velocity components, the vorticity and the pressure gradient. In terms of $v$ the vorticity is the Laplacian of $v$ and the pressure gradient involves the derivatives of $\Delta v$.

Hence we are interested in estimating only the derivatives of $v$ and not $v$ itself. At the same time we would like for these bounds to involve only $\eta_{2}, \epsilon_{1}$, and $\epsilon_{2}$, and not $\eta_{1}$. In order to accomplish this we consider instead of (2.13) the identity

$$
\begin{aligned}
w(P)-w(\bar{P})=-\int_{\omega(r)}[w-w(\bar{P})] & \frac{\partial}{\partial n}(\Delta \Gamma) d s-\int_{\omega(r)} \Delta w \frac{\partial \Gamma}{\partial n} d s \\
& -\int_{\Sigma^{\prime}}\left\{[w-w(\bar{P})] \frac{\partial}{\partial n}(\Delta \Gamma)-\frac{\partial w}{\partial n} \Delta P+\Delta w \frac{\partial \Gamma}{\partial n}-\frac{\partial}{\partial n}(\Delta w) \Gamma\right\} d \sigma,
\end{aligned}
$$

where $\bar{P}$ is the point on $\Sigma$ at which $K\left(r_{0}\right)$ is tangent to $C$. 
For points away from the boundary of $D_{r}$ we differentiate (3.1) carrying out the differentiation of $\Gamma$ on the right. An application of Schwarz's inequality yields the same types of terms as those encountered previously except for the terms $\int_{\omega(r)}\left[w-w(\bar{P})^{2}\right] d s$ and $\int_{\Sigma}[w-w(\bar{P})]^{2} d \sigma$. Using the arguments employed in deriving (2.29) with $w$ replaced by $w-w(\bar{P})$, we may bound the integral over $\omega(r)$ in terms of $\eta_{2}, \epsilon_{1}, \epsilon_{2}$, and $\int_{\Sigma}[w-w(\bar{P})]^{2} d \sigma$. Let us now define $\lambda$ by

$$
\lambda=\min _{\psi} \frac{\int_{0}^{l}\left(\frac{\partial \psi}{\partial \sigma}\right)^{2} d \sigma}{\int_{0}^{l} \psi^{2} d \sigma}
$$

among continuously differentiable functions $\psi$ which vanish at the origin. The number $\lambda$ is the lowest eigenvalue for a vibrating string fixed at the origin and free at $\sigma=l$. Thus

$$
\lambda=\pi^{2} / 4 l^{2} .
$$

We now let $l$ be the shortest distance on $\Sigma$ from $\bar{P}$ to either of the points of intersection of $\omega(r)$ and $\Sigma$, and denote by $L$ the length of $\Sigma$. Thus

$$
l \leq L / 2
$$

and since $w-w(\bar{P})$ is clearly an admissible function $\psi$ in (2.32) - the origin being taken at $\bar{P}$-we obtain using (3.3) and (3.4)

$$
\int_{\Sigma}[w-w(\bar{P})]^{2} d \sigma \leq L^{2} \pi^{-2} \int_{\Sigma}\left(\frac{\partial w}{\partial \sigma}\right)^{2} d \sigma \leq L^{2} \pi^{-2} \eta_{2}
$$

It is thus possible to obtain in $D_{r}$ the following bounds for the velocity components $w_{, x}$ using (2.7), (2.9), (2.30), (3.5), and (2.13):

$$
\left|w_{, x} w_{, x}\right|^{2} \leq C_{1}^{\prime} M_{1}^{1-\delta^{*}} \eta_{2}^{\delta^{*}}+C_{2}^{\prime} M_{2}^{1-\delta^{\prime}}\left\{\kappa_{1} \epsilon_{1}+\kappa_{2} \epsilon_{2}\right\}^{\delta^{\prime}},
$$

where $C_{1}^{\prime}$ and $C_{2}^{\prime}$ are constants and all other terms are the same as those occurring on the right in $(2.30)$. Note that as in [5] the constant $M_{1}$, which is a bound for $|w-w(\bar{P})|$, may be replaced by a constant $L_{1}$, which involves a bound for the kinetic energy in $D$.

\section{References}

[1] J. Hadamard, Le Problème de Cauchy (Paris, 1932).

[2] M. M. Laurentiev, On the Cauchy problem for the Laplace equation, Izvest. Akad. Nauk SSSR, Ser. Mat. 20, 819-842 (1956).

[3] A. E. H. Love, The Mathematical Theory of Elasticity (Dover Publ., New York, N.Y , 1944).

[4] L. M. Milne-Thomson, Theoretical Hydrodynamies (The MacMillan Co., New York, N.Y., 1950).

[5] L. E. Payne, Bounds in the Cauchy problem for the Laplace equation, Archive Rat. Mech. Anal. 5, 35-45 (1960).

[6] L. E. Payne and H. F. Weinberger, New bounds in harmonic and biharmonic problems, J. Math. Phys. 4, 291-307 (1955).

[7] F. Rellich, Darstellung der Eigenwerte von $\Delta u+\lambda u$ durch ein Randintegral, Math. Z. 46, 635-636 (1940).

(Paper 65B2-55) 


\section{Publications of the National Bureau of Standards}

\section{(Including Papers in Outside Journals)}

\section{Selected Abstracts}

On Stokes flow about a torus, W. H. Pell and L. E. Payne, Mathematika $\boldsymbol{\gamma}, 78-92(1960)$.

This is the third of a series of papers on the Stokes (slow viscous) flow about axially symmetric bodies immersed in a uniform flow parallel to the axis of symmetry. The flow itself then has axial symmetry and is amenable to attack by the generalized axially symmetric potential theory of A. Weinstein. In this particular paper the flow in multiple connected regions is considered and, in particular, the problem of flow about a torus is solved. The mathematical problem which characterizes the flow does not have a unique solution. Uniqueness is insured by the physical condition that the pressure be continuous throughout the flow field.

Modern theories of materials, C. Truesdell, Trans. Soc. Rheology IV, 9-22 (1960).

Theoretical work on rheological materials shows three distinct phases:

1. Early work, to 1945 . Simple models, mainly one-dimensional and linear, are constructed by analogy to discrete systems. Various coefficients are named.

2 . Intermediate period, 1945-1955. Ideal materials exhibiting nonlinear response are defined explicitly as exact mathematical realizations of a simple idea. Their properties are studied by means of general theorems and special exact solutions.

3. Current phase, from 1955. The most general constitutive equations consistent with the observed phenomena are determined.

Work of the second and third kinds is summarized, with particular reference to the different roles of methods of invariance.

Open problems are stated.

Graphs of bivariate normal probabilities, M. Zelen and N. C. Severo, Ann. Math. Stat. 31, No. 3, 619-624 (Sept. 1960).

Recently there has been much activity dealing with the tabulation of the bivariate normal probability integral. D. B. Owen [3], [4] has summarized many of the properties of the bivariate normal distribution function and tabulated an auxiliary function which enables one to calculate the bivariate normal probability integral. In addition, the National Bureau of Standards [1] has compiled extensive tables of the bivariate normal integral drawn from the works of K. Pearson, Evelyn Fix and J. Neyman, and H. H. Germond. In this same volume, D. B. Owen has contributed an extensive section on applications.

It is the purpose of this paper to present three charts, which will enable one to easily compute the bivariate normal integral to a maximum error of $10^{-2}$. This should be sufficient for most practical applications. Owen and Wiesen [5] have also presented charts with a similar objective; however, as pointed out below, we believe the charts presented here lend themselves more easily to visual interpolation. Actually the motivation for these charts came from the Owen and Wiesen work.

Comment on a paper of Mori on time-correlation expressions for transport properties, M. S. Green, Phys. Rev. 119, No. 3, 829-830 (Aug. 1960).

An auto-correlation expression given by Mori for the thermal conductivity of a fluid is shown to be only apparently different from an expression previously derived by the author.
The Stokes flow about a spindle, W. H. Pell and L. E. Payne, Quart. Appl. Math. 18, 257-262 (1960).

This is a continuation of earlier work by the same authors. The methods of the generalized axially symmetric potential theory of A. Weinstein and certain representation theorems of L. Payne for the solution of repeated operator equations are applied to the solution of the Stokes (slow) flow about a spindle-shaped body. The stream function of the flow is found and an expression is given for the drag of the body.

Irrational power series, M. Newman, Proc. Am. Math. Soc. 11, 699-802 (Oct, 1960).

It is shown that if $\alpha$ is a real number, $g$ a non-constant polynomial, then

$$
\sum_{n=0}^{\infty} g([n \alpha]) \mathrm{x}^{n}
$$

is a rational function of $\mathrm{x}$ if and only if $\alpha$ is a rational number. The same statement is proved for the function

$$
\sum_{n=0}^{\infty} \mathrm{x}^{[n \alpha]} \text {. }
$$

Topological derivation of the Mayer density series for the pressure of an imperfect gas, M. S. Green, J. Math. Phys. 1, No. 5, 391-394 (Sept.-Oct. 1960).

A new derivation of Mayer's classical density expansion for the pressure of an imperfect gas based on a classification of cluster graphs according to topological criteria is presented. The classification is a generalization of the classification of simple trees into trees with centers and trees with bicenters.

Ensemble method in the theory of irreversibility, R. Zwanzig, J. Chem. Phys. 33, No. 5, 1338-1341 (Nov. 1960).

We describe a new formulation of methods introduced in the theory of irreversibility by Van Hove and Prigogine, with the purpose of making their ideas easier to understand and to apply. The main tool in this reformulation is the use of projection operators in the Hilbert space of Gibbsian ensemble densities. Projection operators are used to separate an ensemble density into a "relevant" part, needed for the calculation of mean values of specified observables, and the remaining "irrelevant" part. The relevant part is shown to satisfy a kinetic equation which is a generalization of Van Hove's "master equation to general order." Diagram summation methods are not used. The formalism is illustrated by a new derivation of the Prigogine-Brout master equation for a classical weakly interacting system.

On the theory of the critical point of a simple fluid, M. S. Green, J. Chem. Phys. 33, No.5, 1403-1409 (Nov. 1950).

The consequences of a new system of integral equations for the theory of the critical point are discussed. Reasons are given for believing that the fundamental assumption of the Ornstein-Zernicke theory about the direct correlation function is incorrect.

\section{List of Titles}

Journal of Research, Section 65A, No. 2, March-April 1961. g0 cents.

Mass spectra of some deuteroethanes, E. I. Quinn and F. L. Mohler.

Heats of hydrolysis and formation of potassium borohydride, W. H. Johnson, R. H. Schumm, I. H. Wilson, and E. J. Prosen. 
Heat of combustion of borazine $\mathrm{B}_{3} \mathrm{~N}_{3} \mathrm{H}_{6}$, M. V. Kilday, W. H. Johnson, and E. J. Prosen.

Thermodynamic properties of thorium dioxide from 298 to $1,200^{\circ}$ K, A. C. Victor and T. B. Douglas.

Calculated energy dissipation distribution in air by fast electrons from a gun source, J. E. Crew.

Vitrons as flow units in alkali silicate binary glasses, L. W. Tilton.

Tetragermanates of strontium, lead, and barium of formula type $\mathrm{AB}_{4} \mathrm{O}_{9}$, C. R. Robbins and E. M. Levin.

Journal of Research, Section 65C, No. 2, April-June 1961. 75 cents.

An experimental study concerning the pressurization and stratification of liquid hydrogen, A. F. Schmidt, J. R. Purcell, W. A. Wilson, and R. V. Smith.

Temperature dependence of elastic constants of some cermet specimens, S. Spinner.

Analog simulation of zone melting, H. L. Mason.

Residual losses in a guard-ring micrometer-electrode holder for solid-disk dielectric specimens, A. H. Scott and W. P. Harris.

A bolometer mount efficiency measurement technique, G. F. Engen.

Telescope for measurement of optic angle of mica, S. Ruthberg.

An automatic fringe counting interferometer for use in the calibration of line scales, H. D. Cook and L. A. Marzetta.

Journal of Research, Section 65D, No. 3, May-June 1961. 70 cents.

Propagation studies using direction-finding techniques, E. C. Hayden.

Diversity effects in long distance high frequency radio pulse propagation, S. A. Bowhill.

Influence of ionospheric conditions on the accuracy of high frequency direction finding, P. J. D. Gething.

Phase difference observations at spaced aerials and their application to direction finding, W. C. Bain.

Research at the National Bureau of Standards applicable to long-distance location and direction-finding problems, R. Silberstein.

Design for spinning goniometer automatic direction finding, W. J. Lindsay and D. S. Heim.

Resolution characteristics of correlation arrays, I. W. Linder.

Instrumentation for propagation and direction-finding measurements, E. C. Hayden.

Brooke variance classification system for DF bearings, E. M. L. Beale.

Estimation of variances of position lines from fixes with unknown target positions, E. M. L. Beale.

Statistics of a radio wave diffracted by a random ionosphere, S. A. Bowhill.

Space analysis of radio signals, J. B. Smyth.

Effect of receiver bandwidth on the amplitude distribution of VLF atmospheric noise, F. F. Fulton, Jr.

Excitation of VLF and ELF radio waves by a horizontal magnetic dipole, J. Galejs.

Journal of Research, Section 65D, No. 2, March-April 1961. 70 cents.

Ionospheric motions observed with high-frequency backscatter sounders, L. H. Tveten.

Relationship between red auroral ares and ionospheric recombination, G. A. M. King and F. E. Roach.

Fresnel region fields of circular aperture antennas, MingKuei $\mathrm{Hu}$.

Free-balloon borne meteorological refractometer, J. F. Theisen and E. E. Gossard.

Weather and reception level on a troposphere link - annual and short-term correlations, L. G. Abraham, Jr., and J. A. Bradshaw.

Initial results of a new technique for investigating sferic activity, G. Hefley, R. H. Doherty, and R. F. Linfield.

Effect of antenna radiation angles upon $\mathrm{HF}$ radio signals propagated over long distances, W. F. Utlaut.

Graphic determination of radio ray bending in an exponential atmosphere, C. F. Pappas, L. E. Vogler, and P. L. Rice.
A formula for radio ray refraction in an exponential atmosphere, G. D. Thayer.

The impedance of a monopole antenna with a circular conducting-disk ground system on the surface of a lossy half-space, S. W. Maley and R. J. King.

Radio-wave propagation in the earth's crust, H. A. Wheeler.

Climatic charts and data of the radio refractive index for the United States and the world, B. R. Bean, J. D. Horn, and A. M. Ozanich, Jr., NBS Mono. 22 (1960) \$2.

Amplitude-probability distributions for atmospheric radio noise, W. Q. Crichlow, A. D. Spaulding, C. J. Roubique, and R. T. Disney, NBS Mono. 23 (1960) 20 cents.

Units of weight and measure (United States customary and metric) definitions and tables of equivalents, NBS Misc. Publ. 233 (1960) 40 cents.

Report of the $45 \mathrm{th}$ national conference on weights and measures 1960, NBS Misc. Publ. 235 (1960) 75 cents.

Standard frequencies and time signals from NBS stations WWV and WWVH, NBS Misc. Publ. 236 (1960) 10 cents.

Research highlights of the National Bureau of Standards, Annual Report, Fiscal year 1960, NBS Misc. Publ. 237 (1960) 65 cents.

Building code requirements for reinforced masonry, NBS Handb. H74 (1960) 15 cents.

Report of the International Commission on radiological units and measurements (ICRU) 1959, NBS Handb. H78 (1961) 65 cents.

Supplementary world map of F2 critical frequencies and maximum usable frequency factors, D. H. Zacharisen, NBS TN2-2 (PB151361-2) (1960) \$3.50.

Quarterly radio noise data-December, January, February 1959-60, W. Q. Crichlow, R. D. Disney, and M. A. Jenkins, NBS TN18-5 (PB151377-5) (1960) \$1.75

Quarterly radio noise data-March, April, May 1960, W. Q Crichlow, R. D. Disney, and M. A. Jenkins, NBS TN18-6 (PB151377) (1960) \$1.75.

Quarterly radio noise data-June, July, August 1960, W. Q. Crichlow, R. D. Disney, and M. A. Jenkins, NBS TN18-7 (PB151377-7) (1960) $\$ 1.75$.

On the nature of the crystal field approximation, H. Goldberg and C. Herzfeld, NBS TN67 (PB161568) (1960) $\$ 2.50$.

Vapor pressures of organic compounds in the range below one millimeter of mercury, E. E. Hughes and S. G. Lias, NBS TN70 (PB161571) (1960) 75 cents.

Some experiments on the deposition of gases at $4.2^{\circ} \mathrm{K}, \mathrm{T}$. Baurer, NBS TN73 (PB161574) (1960) \$1.00.

Scattering of cobalt-60 gamma radiation in air ducts, C. Eisenhauer, NBS TN74 (PB161575) (1960) 75 cents.

Soviet research in field electron and ion emission, 1955-1959; an annotated bibliography, T. W. Marton and R. Klein, NBS TN75 (PB161576) (1960) \$1.25.

Oblique incidence receiving antenna array for a relative ionospheric opacity meter, A. C. Wilson, NBS TN78 (PB161579) (1960) 50 cents.

$\mathrm{VHF}$ radio propagation data for Cedar Rapids-Sterling, Anchorage-Barrow, and Fargo-Churchill test paths April 1951 through June 1958, G. R. Sugar and K. W. Sullivan, NBS TN79 (PB161580) (1960) \$4.00.

Bibliography of tropospheric radio wave scattering, R. L. Abbott, NBS TN80 (PB161581) (1960) \$2.25

A survey of spread-F, F. N. Glover, NBS TN82 (PB161583) (1960) $\$ 1.75$.

The NBS meteor-burst propagation project-a progress report, C. E. Hornback, L. D. Breyfogle, and G. R. Sugar, NBS TN86 (PB161587) (1960) \$1.25.

A theoretical study of sporadic- $E$ structure in the light of radio measurements, $\mathrm{K}$. Tao, NBS TN87 (PB161588) (1961) $\$ 1.25$

The dynamic compressibility of a rubber-sulfate vulcanizate and its relation to free volume, J. E. McKinney, H. V. Belcher, and R. S. Marvin, Trans. Soc. Rheology IV, 347-362 (1960).

Procedure for the determination of the noble metal content of dental gold alloys, H. J. Caul, W. S. Clabaugh and M. E. Susa, J. Am. Dental Assoc. 61, No. 4, 339-443 (Oct. 1960) 
Rapid frequency analysis of fading radio signals, J. N. Watts and K. Davies, J. Geophys. Research 65, No. 8, 2295-2301 (Aug. 1960).

Reply to criticisms concerning Ireland contained in article "Basic research in Europe," D. M. Gates, Science 128, No. 3318, 1-9 (Aug. 1, 1958).

Thermal voltage converters for accurate voltage measurements to 30 megacycles per second, F. L. Hernach and E. S. Williams, Commun. Electron. AIEE, No. 49, 200-206 (July 1960).

Use of the incoherent scatter technique to obtain ionospheric temperatures, T. E. VanZandt and K. L. Bowles, J. Geophys. Research 65, 2627-2628 (Sept. 1960).

Highlights of the consultative committee on international radio (CCIR) activities in the field of radio propagation, J. W. Herbstreit, 9th Plenary Assembly of CCIR Proc. IRE 48, No. 1, 45-53 (Jan. 1960).

Temperature stratification in a non-venting liquid helium dewar, L. E. Scott, R. F. Robbins, and B. W. Birmingham, Proc. 1959 Cryogenic Engr. Conf. 5 (Sept. 2-4, 1959).

A microwave impedance meter capable of high accuracy, R. W. Beatty, IRE Trans. Microwave Theory and Tech. MTT-8, No. 4 (July 1960).

Variational treatment of electron-hydrogen atom elastic scattering, S. Geltman, Phys. Rev. 119, No. 4, 1283-1290 (Aug. 1960).

Relaxation processes in multistate systems, K. E. Shuler, Phys. Fluids 2, No. 4, 442-448 (July-August 1959).

Comment on models of the ionosphere above $h_{\max } F 2, \mathrm{~J} . \mathrm{W}$ Wright, J. Geophys. Research 65, 2595-2596 (Sept. 1960).

Some magnetoionic phenomena of the Arctic E-region, J. W. Wright, J. Atmospheric and Terrest. Phys. 18, 276-289 (Aug. 1960).

Tables of thermodynamic and transport properties of air, argon, carbon dioxide, carbon monoxide, ${ }^{\text {hydrogen, nitro- }}$ gen, oxygen and steam, J. Hilsenrath, C. W. Beckett, W. S. Benedict, L. Fano, H. J. Hoge, J. F. Masi, R. L. Nuttall, Y. S. Touloukian, and H. W. Woolley, 478 pages (Pergamon Press, Oxford, London, New York, and Paris, $1960)$

Methods of nuclear orientation, E. Ambler, Progress in Cryogenics 2, 235-280 (Haywood \& Co., Ltd., London, England, 1960).

A conference on the propagation of ELF electromagnetic waves, J. R. Wait, Proc. IRE 48, 1648-1649 (Sept. 1960).

The strength of ten structural adhesives at temperatures down to minus 424 F., W. M. Frost, Proc. 1959 Cryogenic Engr. Conf. 5, 375-384 (Sept. 2-4, 1959).

Possibility of detecting ionospheric drifts from the occurrence of spread $F$ echoes at low latitudes, R. W. Knecht, Nature Letter 187, 927 (Sept. 1960).

A compilation and correlation of the PVT data of normal hydrogen from saturated liquid to 80 degree K., R. B. Steward and V. J. Johnson, Proc. 1959 Cryogenic Engr. Conf. 5, 548-556 (Sept. 2-4, 1959).

Pressure dependence of rotationally perturbed lines in the ultraviolet band spectrum of $\mathrm{CN}, \mathrm{H}$. P. Broida and S. Golden, Can. J. Chem. 38, 1666-1677 (1960).

Neighbor interactions and internal rotations in polymer molecules. IV. Solvent effect on internal rotations, S. Lifson and I. Openheim, J. Chem. Phys. 33, No. 1, 109-115 (July 1960)

Improvements in radio propagation prediction service, W. E. Chadwick, Elec. Engr. 1-4 (Sept. 1960).

Carbon resistance thermometry with mixed dc and rf currents, J. J. Gniewek and R. J. Corruccini, Rev. Sci. Inst. 31, No. 8, 899-900 (Aug. 1960).

Some SEAC computations of subsonic flows, P. Davis and P. Rabinowitz, book, Bergmann's Linear Integral Operator Method in the Theory of Compressible Fluid Flow, by M. Z. Krzywoblocki, p. 148-172 (Wien, Springer-Verlag, Berlin, Germany, 1960).

Spatial distribution of energy dissipated by fallout beta rays, A. E. Boyd and E. E. Morris, Health Phys. 2, 321-325 (1960).

Maser frequency stability, R. C. Mockler and J. A. Barnes, Proc. 13. Annual Frequency Control Symp.,583 -595 (May $12-14,1959)$

Acid-base equilibria in benzene at three temperatures. The comparative reactivities of a phenolic acid and a carboxylic acid with triethylamine with 1,3-diphenylguanidine, M. M. Davis and M. Paabo, J. Am. Chem. Soc. 82, 5081-5084 (1960).

Absolute isotopic abundance ratio and the atomic weight of silver, W. R. Shields, D. N. Craig, and V. H. Dibeler, J. Am. Chem. Soc. 82, 5033-5036 (1960).

Some mechanical properties of magnesium alloys at low temperatures, R. P. Reed, R. P. Mikesell and R. L. Greeson, Proc. 1959, Cryogenic Engr. Conf. 5, 397-405 (Sept. 2-4, 1959).

Electron spin resonance studies of free radicals in irradiated materials, L. A. Wall, Symp. on Materials in Nuclear Application, Am. Soc. Testing Materials Spec. Tech. Publ. No. 276, 208-223 (1959).

Flexural strength of specimens prepared from several uranium dioxide powders; its dependency on porosity and grain size and the influence of additions of titania, F. P. Knudsen, H. S. Parker, and M. D. Burdick, J. Am. Ceram. Soc. 43, No. 12, 641-647 (Dec. 1960).

Green and purple sulfur: Electron-spin resonance studies, H. E. Radford and F. O. Rice, J. Chem. Phys. 33, No. 3, 774-776 (Sept. 1960).

Correlation of an auroral arc with a subvisible monochromatic $6300 \mathrm{~A}$ are with outer-zone radiation on November 28, 1959 , B. J. O'Brien, J. A. Van Allen, F. E. Roach, and C. W' Gartlein, J. Geophys. Research 65, No. 9, 2759-2766 (Sept. 1960).

Report on the standardization of $p \mathrm{H}$ and related terminology, R. G. Bates and E. A. Guggenheim, Intern. Union Pure and Appl. Chem. 1, No. 1, 163-168 (1960).

Spectrum of $\operatorname{ReF}_{6}$, J. C. Eisenstein, J. Chem. Phys. 33, No. 5, 1530-1531 (Nov. 1960).

Regulated power supply for instruments, W. V. Loebenstein, Electronics 33, No. 48, 132 (Nov. 1960).

Effect of structure on the spectra emitted by solid nitrogen during electron bombardment, L. J. Schoen and H. P. Broida, J. Mol. Spectroscopy 5, No. 5, 416-419 (Nov. 1960).

Chemical reactions of free radicals at low temperatures, R. A. Ruehrwein, J. S. Hashman and J. W. Edwards, J. Phys. Chem. 64, 1317-1322 (1960).

Experimental investigation of creep deflection of extruded and riveted I-beams, L. Mordfin and N. Halsey, NASA Tech. Note D-662 (Dec. 1960).

Spatial distribution of energy dissipated by fallout-rays, A. E. Boyd and E. E. Morris, Health Phys. 2, 321-325 (1959).

Megaroentgen dosimetry employing photographic film without processing, W. L. McLaughlin, Radiation Research 13, No. 4, 594-609 (Oct. 1960).

The reaction of hydrogen atoms with solid propene at low temperatures, R. Klein, M. D. Scheer and J. G. Waller, J. Phys. Chem. 64, 1247-1250 (1960).

The measurement of thermal conductivity, D. C. Ginnings, book, Thermoelectric Materials and Devices, edited by I. B. Cadoff and E. Miller, Chap. 8, 113-132 (Reinhold Publishing Corporation, New York, N.Y., 1960).

Programming for a closed-loop, manned-machine combined system, D. C. Friedman, Proc. Combined Analog-Digital Computer Systems Symp., December 16 and 17, 1960, Philadelphia, Pa., 12th Article (Dec. 1960).

Analytical study of creep deflection of structural beams, L. Mordfin, NASA Tech. Note D-661 (Dec. 1960).

Evaluation of ball-bearing separator materials operating submerged in liquid nitrogen, W. A. Wilson, K. B. Martin, J. A. Brennan, and B. W. Birmingham, ASLE. ASME Lubrication Conf., October $17-19,1960$ (Boston, Mass.), Am. Soc. Lubrication Engrs. Preprint No. 60 LC-4 (1960).

Microwave spectrum, structure, and dipole moment of propane, D. R. Lide, Jr., J. Chem. Phys. 33, No. 5, 15141518 (Nov. 1960).

Surface area determination of kaolinite using glycerol adsorption, K. H. Woodside and W. C. Ormsby, J. Am. Ceram. Soc. 43, No. 12, 671-672 (Dec. 1960).

Magnetic resonance determination of the nuclear moment of tantalum-181 in $\mathrm{KTaO}_{3}$, L. H. Bennett and J. I. Budnick, Phys. Rev. 120, No. 5, 1812-1815 (Dec. 1, 1960).

Aircraft storage batteries, W. J. Hamer, AIEE and Am. Inst. Elec. Engr. Trans. 79, Pt. II, 1-11 (Sept. 1960). 
Electrodeless passage of direct current through an electrolyte, A. Brenner, J. Electrochem. Soc. 10\%, No. 12, 968-973 (Dec. 1960)

Preparation and thermal stability of tetrakis-(pentafluorophenyl)-silane and tris-(pentafluorophenyl)-phosphine, L. A. Wall, R. E. Donadio, and W. J. Pummer, J. Am. Chem. Soc. 32, No. 13, 4846-4852 (Sept. 1960).

Stress-strain relationships in yarns subjected to rapid impact loading. Part VI: Velocities of strain waves resulting from impact, J. C. Smith, J. M. Blanford, and H. F. Schiefer, Textile Research J. 30, No. 10, 752-760 (Oct. 1960).

The centennial of spectrochemistry, W. F. Meggers, J. L. Tech. J. Optical Soc. Am. 50, No. 11, 1035-1038 (Nov. 1960)

Rate of reaction of nitrogen atoms with ethylene, J. T. Herron, J. Chem. Phys. 33, No. 4, 1273-1274 (Oct, 1960).

Structure of the isobutane molecule; change of dipole moment on isotopic substitution, D. R. Lide, Jr., J. Chem. Phys. 33, No. 5, 1519-1522 (Nov. 1960).

Condensation coefficient of arsenic trioxide glass, A. B. Bestul and D. H. Blackburn, J. Chem. Phys. 33, No. 4, 1274-1275 (Oct. 1960).

A comparison of atomic beam frequency standards, R. E. Beehler, R. C. Mockler, and C. S. Snider, Nature 18;, No. 4738, 681-682 (Aug. 20, 1960).

The compound $\mathrm{BaTiGe}_{3} \mathrm{O}_{9}$, C. R. Robbins, J. Am. Ceram. Soc. 43, No. 11, 610 (Nov. 1960).

Some ceramic dielectrics with a very low temperature coefficient of capacitance, S. Marzullo and E. N. Bunting, J. Am. Ceram. Soc. 43, No. 11, 609 (Nov. 1960).

Vibration-rotation bands of $\mathrm{N}_{2} \mathrm{O}$, E. D. Tidwell and E. K. Plyler, J. Op. Soc. Am. 50, No. 12, 717-720 (Dec. 1960).

Nuclear magnetic resonance in tantalum metal, J. I. Budnick and L. H. Bennett, J. Phys. Chem. Solids 16, No. 1/2, 37-38 (Jan. 12, 1960).

The sample, the procedure, and the laboratory, W. J. Youden, Anal. Chem. 32, No. 13, 23A-37A (Dec. 1960)

The relation of $h_{\max } F 2$ to $M(3000) F 2$ and $H_{q} F 2, J . W$. Wright and R. E. McDuffie, J. Radio Research Lab. $\boldsymbol{g}$, No. 32, 409-420 (July 1960).

8-quinolinol precipitation of the elements, J. I. Hoffman, Chem. Anal. 49, No. 4, 126 (Dec. 1960).

Dipole moments of hydrocarbons, D. R. Lide, Jr., J. Chem Phys. 33, No. 6, 1879 (Dec. 1960).

Collection of ions produced by alpha particles in air, Z. Bay and H. H. Seliger, Phys. Rev. 120, No. 1, 141-143 (Oct. 1, 1960).

A test of a procedure for easy estimation of representative monthly electron density profiles for the ionosphere, J. W Wright, J. Geophys. Research 65, No. 10, 3215-3217 (Oct. 1960).

A method for efficiently providing low temperature liquids on a large scale to an accelerated experimental program, A. K. Stober, Proc. 10th Intern. Congress of Refrigeration, Copenhagen, Denmark 1, No. 1, 17-18 (Pergamon Press Ltd., London, England, 1959).
Seasonal variations in the twilight enhancement of [OI] 5577 , L. R. Megill, P. M. Jamnick and J. E. Cruz, J. Atmospheric and Terrest. Phys. 18, 309-314 (Aug. 1960).

Synthetic mica, A. V. Valkenburg, Book, Encyclopedia of Chemical Technology, p. 480-487 (Interscience Encyclopedia, Inc., New York, N.Y., 1960).

Nonresonant microwave absorption and relaxation frequency at elevated pressures, A. A. Maryott and G. Birnbaum, J. Phys. Chem. 64, No. 11, 1778-1780 (Nov. 1960).

Precipitation of the elements on addition of aqueous ammonia to their clear solution, J. I. Hoffman, Chem.-Anal. 49, No. 3, 94 (Sept. 1960).

Low scatter high current gas target for D-D neutrons, A. C. B. Richardson, Rev. Sci. Inst. 31, No. 11, 1202-1203 (Nov. 1960).

The heat of combustion of dicyanoacetylene, G. T. Armstrong and S. Marantz, J. Phys. Chem. 64, 1776-1777 (1960).

Evaluation of resistance strain gages at elevated temperatures, R. L. Bloss, Am. Soc. Testing Materials Proc. 1, No. 1, 9-15 (Jan. 1961)

Errors in dielectric measurements due to a sample insertion hole in a cavity, A. J. Estine, and H. E. Bussey, IRE Trans. Microwave Theory and Tech. MTT-8, No, 6, 650-653 (Nov. 1960).

Determination of pentosans. Interlaboratory comparison of the aniline acetate, orcinol, and bromination methods, W. K. Wilson and J. Mandel, Tappi 43, No. 12, 998-1004 (Dec. 1960).

A research for geomagnetic singular days, C. Warwick, T. Pohrte, and N. MacDonald, J. Geophys. Research 65, No. 9, 3013-3015 (Sept. 1960).

Letter to editor of Revue Des Materiaux (first), E. S. Newman and H. A. Berman, Revue Des Materiaux C, No. 540, 231 (Dec. 1, 1960).

Geomagnetic storms and the space around the earth, S. Chapman, Nature 187, No. 4740, 824-827 (Sept. 3, 1960). Some implications of slant- $E_{s}$, E. K. Smith and R. W. Knecht, Polar Atmosphere Symp., Part 2, Ionospheric Section, Oslo, Norway, July 2-8, 1956, p. 195-204 (Perga. mon Press, Inc., New York, N.Y., 1957).

Mismatch errors in microwave phase shift measurements, G. E. Schafer, IRE Trans. Microwave Theory and Tech. MTT-8, No. 6, 617-622 (Nov. 1960).

Residual arc spectra of seventy elements diluted in copper, C. H. Corliss, W. F. Meggers and B. F. Scribner, book, Colloquim Spectroscopicum Internationale VIII, 119-121 (1959).

The rotational constants of hydrogen chloride, E. K. Plyler and E. D. Tidwell, Z. Elektrochem. 64, No. 5, 717-720 (Feb. 1960).

Publications for which a price is indicated (except for Technical Notes) are available only from the Superintendent of Documents, U.S. Government Printing Office, Washington 25, D.C. (foreign po stage, one-fourth additional). Reprints from outside journals and the NBS Journal of Research may often be obtained directly from the authors. 